To appear in Nature

\title{
The Cosmic Microwave Background temperature at a redshift of 2.33771
}

\author{
R. Srianand ${ }^{1}$, P. Petitjean ${ }^{2,3}$, C. Ledoux ${ }^{4}$ \\ 1) IUCAA, Post Bag 4, Ganeshkhind, Pune 411 007, India \\ 2) Institut d'Astrophysique de Paris - CNRS, 98bis Boulevard Arago, F-75014 Paris, France \\ 3) CNRS 173 - DAEC, Observatoire de Paris-Meudon, F-92195 Meudon Cedex, France \\ 4) European Southern Observatory, Karl Schwarzschild Strasse 2, D-85748 Garching bei \\ München, Germany
}


The Cosmic Microwave Background radiation is a fundamental prediction of Hot Big Bang cosmology. The temperature of its black-body spectrum has been measured at the present time, $T_{\mathrm{CMBR}, 0}=\mathbf{2 . 7 2 6} \pm \mathbf{0 . 0 1 0} \mathrm{K}$, and is predicted to have been higher in the past. At earlier time, the temperature can be measured, in principle, using the excitation of atomic fine structure levels by the radiation field. All previous measurements however give only upper limits as they assume that no other significant source of excitation is present. Here we report the detection of absorption from the first and second fine-structure levels of neutral carbon atoms in an isolated remote cloud at a redshift of 2.33771. In addition, the unusual detection of molecular hydrogen in several rotational levels and the presence of ionized carbon in its excited fine structure level make the absorption system unique to constrain, directly from observation, the different excitation processes at play. It is shown for the first time that the cosmic radiation was warmer in the past. We find $6.0<T_{\mathrm{CMBR}}<14 \mathrm{~K}$ at $z=2.33771$ when $9.1 \mathrm{~K}$ is expected in the Hot Big Bang cosmology.

One of the firm predictions of standard Big Bang model is the existence of relic radiation from the hot phase the Universe has experienced at early timest. The cosmic microwave background radiation (CMBR) has been discovered serendipitously by Penzias \& Wilsont in 1964. The fact that its spectrum follows with remarkable precision a Planckian distribution over several decades in frequency is a strong argument in favor of the Hot Big Bang cosmology. However the presence of the radiation at earlier time has never been proven directly. The temperature of its black-body spectrum is predicted to increase linearly with redshift $T_{\mathrm{CMBR}}(z)=T_{\mathrm{CMBR}}(0) \times(1+z)$ and its local value has been determined very accurately by the Cosmic Background Explorer (COBE) $T_{\mathrm{CMBR}}(0)=2.726 \pm 0.010 \mathrm{~K}$ 3. Detecting the presence of relic radiation at earlier epochs and confirming the well defined temperature evolution is therefore a crucial test for cosmology. To perform this test, one can use the possibility that excited fine structure levels of the ground-state of atomic species can be partly populated by the Cosmic Microwave Background Radiation when the energy separation of the levels is similar to the energy at the peak of the radiation energy 
distribution.

The relative populations of excited levels can be measured from the absorption lines seen in the

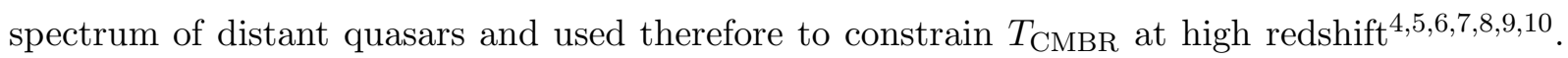
The expected values make neutral carbon, $\mathrm{C}^{0}$, particularly well suited for this purpose. The ground term is split into three levels $(J=0,1,2)$ with $J=0-1$ and $J=1-2$ energy separations $k T$ corresponding to, respectively, $T=23.6$ and $38.9 \mathrm{~K}$. However the fine structure levels can also be excited by collisions (mostly with electrons and hydrogen) and by UV pumping and following cascades. The ionization potential of neutral carbon, $11 \mathrm{eV}$, is below the ionization potential of

hydrogen, $13.6 \mathrm{eV}$, and $\mathrm{C}^{0}$ can only be seen in dense, neutral and highly shielded gas 9 . Therefore, excitation by collisions cannot be neglected.

To constrain $T_{\mathrm{CMBR}}$, the kinetic temperature, the particle density of the gas and the local UV radiation field must be known. As it is very difficult to disentangle these various excitation processes, all measurements up to now have lead to upper limits on $T_{\mathrm{CMBR}}$ only.

Our measurement has been obtained in a unique absorption system where absorption lines of neutral carbon in the three fine-structure level of the ground term, $\mathrm{C}^{0}, \mathrm{C}^{0 *}, \mathrm{C}^{0 * *}$ are observed together with absorption lines of singly ionized carbon in its excited fine-structure level, $\mathrm{C}^{+*}$, and absorption lines of molecular hydrogen $\left(\mathrm{H}_{2}\right)$ in the $J=0$ to 5 rotational levels. The population and depopulation of the first excited rotational level of $\mathrm{H}_{2}(J=1)$ from and to the ground state $(J=0)$ is controlled by thermal collisions. Therefore the excitation temperature $T_{01}$ is approximately equal to the kinetic temperature. The fine structure upper level of the $\mathrm{C}^{+}$ground-state doublet is mostly populated by collisions and depopulated by radiative decay. Therefore, once the temperature is known, the particle density can be derived from the $\mathrm{C}^{+*} / \mathrm{C}^{+}$ ratio. Finally, the UV radiation flux can be constrained from the populations of the $\mathrm{J}=4$ and 5 $\mathrm{H}_{2}$ rotational levels.

\section{Observations}

We have used the Ultra-violet and Visible Echelle Spectrograph (UVES) 11 mounted on the 
ESO KUEYEN $8.2 \mathrm{~m}$ telescope at the Paranal observatory on April 5 and 7, 2000 to obtain a high-spectral resolution spectrum of the $z_{\mathrm{em}}=2.57$ and $m_{\mathrm{V}}=18.4$ quasar PKS $1232+0815$. Standard settings have been used in both arms of the spectrograph. Wavelength ranges were 3290-4519 $\AA$ in the blue; 4623-5594 and 5670-6652 $\AA$ for the red chips. The slit width was 1 arcsec and the CCDs were binned $2 \times 2$ resulting in a resolution, $\Delta \lambda / \lambda$, of $\sim 45000$. The exposure time was 3 hours in seeing conditions better than 0.8 arcsec full width at half maximum. The data were reduced using the UVES pipeline in an interactive mode. The pipeline is a set of procedures implemented in a dedicated context of MIDAS, the ESO data reduction package. The main characteristics of it is to perform a precise inter-order background subtraction for science frames and master flat-fields, and to allow for an optimal extraction of the object signal rejecting cosmic ray impacts and performing sky-subtraction at the same time. The reduction is checked step by step. Wavelengths are corrected to vacuum-heliocentric values and individual 1D spectra are combined together. This resulted in a S/N ratio per pixel of 10 around $3700 \AA$ and 20 around $6000 \AA$. Typical errors in the wavelength calibration is $\sim 0.5 \mathrm{~km} \mathrm{~s}^{-1}$.

\section{Fit to the lines, metallicity and molecular content}

This is the first time $\mathrm{C}^{0}, \mathrm{C}^{0 *}$ and $\mathrm{C}^{0 * *}$ absorption lines are detected at high redshift (see Fig. 1). The detection of these three species at $z_{\mathrm{abs}}=2.33771$ is confirmed by the presence of several transitions. $\mathrm{C}^{0}$ absorption lines with rest wavelengths 1139.79, 1157.91, 1260.73, 1560.31 and $1656.92 \AA, \mathrm{C}^{0 *}$ absorption lines with rest wavelengths 1194.40, 1279.89, 1329.09, 1329.10, 1329.12, $1560.68,1560.71,1656.27,1657.38 \AA$ and 1657.91 and $\mathrm{C}^{0 * *}$ lines at 1657.00 and $1658.12 \AA$ are clearly detected and are free from any blending with absorption due to other systems. We use the oscillator strengths given by Welty et al.12 and standard Voigt profile fitting to determine the amount of matter lying on the line of sight and characterized by column densities, $N$, and

the Doppler parameters, $b=\sqrt{b_{\mathrm{th}}^{2}+b_{\mathrm{turb}}^{2}}$ where $b_{\mathrm{th}}=\sqrt{2 K T / m}$ is the thermal broadening due to the temperature of the gas, with $T$ the kinetic temperature, $K$ the Boltzman constant and $m$ the mass of the particle and $b_{\text {turb }}$ is the turbulent broadening due to macroscopic motions of the gas. It can be seen in Figs. 11 and 2 that the system is dominated by a single and well defined 
component. We consistently impose the same $b$ value to this component for all species and obtain a best fit for $b=1.7 \pm 0.1 \mathrm{kms}^{-1}$. This value is small compared to the spectral resolution of the data but is ascertained by the relative optical depths in the numerous lines with very different oscillator strengths. Results are given in Table 1 and the fit to a portion of the spectrum is shown in the bottom panel of Fig. 1 and in Fig. 2.

The neutral hydrogen column density in the system, $\log N\left(\mathrm{H}^{0}\right)=20.90 \pm 0.10$, has been obtained by fitting the damped Lyman- $\alpha$ line. At such a high column density, hydrogen is mostly neutral and the elements of interest here, iron, magnesium, silicon and carbon are mainly in the singly ionized state. Column densities are derived from simultaneous Voigt profile fitting of all available absorption lines using the same Doppler parameter for all species and the same column density for each species. We concentrate however on weak lines as $\mathrm{Fe}^{+} \lambda \lambda \lambda 1125,1608,1611, \mathrm{Si}^{+} \lambda 1808$ or $\mathrm{Mg}^{+} \lambda 1239$ because they are optically thin. Indeed in this case the strength of the line does not depend on the Doppler parameter and directly gives the column density. Profiles of a few transitions are shown on Fig. 2 and results are given in Table 1. The $\alpha$-chain elements magnesium and silicon have similar abundances, $Z(\mathrm{X})=N(\mathrm{X}) / N(\mathrm{H})$, relative to solar within the measurement uncertainties, $\log Z / Z_{\odot} \sim-1.2$. However we note that, relative to what is seen in the sun, iron is underabundant by about a factor of 5 compared to silicon and nickel is slightly underabundant compared to iron. If these differences are due to iron and nickel being depleted into dust-grains, the depletion is of the same order as what is observed in warm halo gas in our Galaxy 4 . All this suggests that the system is very much like typical damped Lyman- $\alpha$ systems, with metallicity about one tenth of solar and small depletion of iron type element into dust 8, th.

Our echelle data confirms the presence of molecular hydrogen first detected by Ge and Bechtold $\mathrm{E}$. At the resolution of the spectrum, most of the $\mathrm{H}_{2}$ lines are free from blending. The wide wavelength coverage of the spectrum implies that absorption lines from various Lyman-bands are seen. Column densities of $\mathrm{H}_{2}$ in different rotational $J$ levels are obtained by simultaneously fitting various Lyman-bands which are free from contamination due to intervening Lyman- $\alpha$ absorption from the diffuse intergalactic medium. Absorption profiles corresponding to transitions from $J>1$ 
rotational levels are consistent with a single component at the same redshift $z_{\mathrm{abs}}=2.33771$ as the $\mathrm{C}^{0}$ lines. Lines from the $J=0$ and 1 levels are broader and most of the absorption features have similar profiles suggesting the presence of several components. We obtain a best fit to these absorption lines with four components. Results of Voigt profile fitting are given in Table 2. The total $\mathrm{H}_{2}$ column density is $N\left(\mathrm{H}_{2}\right)=1.52 \times 10^{17} \mathrm{~cm}^{-2}$ and the molecular fraction is $f=$ $2 N\left(\mathrm{H}_{2}\right) /\left(2 N\left(\mathrm{H}_{2}\right)+N\left(\mathrm{H}^{0}\right)\right)=3.8 \times 10^{-4}$ which is about two orders of magnitude less than what was derived from low dispersion data.

\section{Physical conditions in the gas}

In molecular clouds, the kinetic temperature of the gas is given by the excitation temperature, $T_{01}$, measured between the $J=0$ and 1 levels. For the components at $z_{\text {abs }}=2.33735,2.33754$ and 2.33793 we find $T_{01}=74 \pm 7,64 \pm 7$ and $66 \pm 10 \mathrm{~K}$ respectively. None of these components show detectable absorption due to $\mathrm{C}^{0}$. The characteristics of the gas are very similar to what has been measured recently in diffuse gas in the halo of our Galaxy 7 . For the $z_{\mathrm{abs}}=2.33771$ cloud, $T_{\text {kin }}=T_{01}=185 \pm 100 \mathrm{~K}$. The excitation temperature for other levels, $J=2$ to 5 , is close to $<T_{e x}>=400 \mathrm{~K}$. This means that processes other than collisions are at play in determining the relative populations of the different levels. Indeed, the $J=4$ and 5 levels can be populated by cascades following UV-pumping and $\mathrm{H}_{2}$ formation. We can evaluate the UV-pumping rate from $J=0$ to $J=4, \beta(0)$, using the simple model of Jura 18 and assuming that molecules are formed on dust grains with formation rate $R$,

$$
p_{4,0} \beta(0) n\left(\mathrm{H}_{2}, J=0\right)+0.19 R n(H) n=A(4 \rightarrow 2) n\left(\mathrm{H}_{2}, J=4\right)
$$

where $n=n(H)+2 n\left(\mathrm{H}_{2}\right), p_{4,0}=0.26$ is the pumping efficiency from level $J=0$ to level $J=4$. We use the spontaneous transition probabilities $A(4 \rightarrow 2)=2.8 \times 10^{-9} \mathrm{~s}^{-1}$ as given by Dalgarno \& Wright 19 . Scaling the formation rate of $\mathrm{H}_{2}$ in our Galaxy $\left(R \sim 3 \times 10^{-17} \mathrm{~s}^{-1} \mathrm{~cm}^{3}\right)$ with the dust to hydrogen ratio measured in the system $\left(\leq 10^{-1.3}\right.$ the Galactic one $)$ it is easily seen that populating the $J=4$ level following formation of a molecule on dust grain is negligible for densities less than $5 \times 10^{3} \mathrm{~cm}^{-3}$. We estimate the density from the excitation of $\mathrm{C}^{+}$to be at 
least a factor of hundred less than this value (see below). Moreover, for such large densities, the $J=0$ and $J=2$ levels as well as the $J=1$ and $J=3$ levels should be in thermal equilibrium which is not the case 20 . We therefore can neglect populating of $J=4$ and 5 levels by cascades after the formation of a molecule. We finally derive from Equation 1 that the photo-absorption rate in the Lyman and Werner bands in the surface of the cloud is of the order of $2 \times 10^{-10} \mathrm{~s}^{-1}$ which is quite modest and similar to that observed along sight-lines in our Galaxy 18.

The excited level of the $\mathrm{C}^{+}$ground-state term is populated by collisions with electrons and hydrogen atoms21. Electrons cannot be neglected because the corresponding collisional cross-section is large. To estimate the electron density we use the ratio $N\left(\mathrm{Mg}^{+}\right) / N\left(\mathrm{Mg}^{0}\right)=178$ which is fairly well determined from the detection of weak lines $\mathrm{Mg}^{0} \lambda \lambda 1737,1827$ and $\mathrm{Mg}^{+} \lambda 1239$. As we have shown above that the ionizing flux in the cloud is similar to that observed in our Galaxy we can estimate the electron density by equating the Galactic ionizing rate of $\mathrm{Mg}^{0}$ to the recombination rate of $\mathrm{Mg}^{+}$目. We find $n_{\mathrm{e}} \sim 0.02 \mathrm{~cm}^{-3}$. Note that the electronic density cannot be much smaller than this value because, from the excitation of the $J=4$ and $5 \mathrm{H}_{2}$ levels, we know that the ionizing flux is not smaller than the Galactic value.

Then, the hydrogen density can be derived from the $N\left(\mathrm{C}^{+*}\right) / N\left(\mathrm{C}^{+}\right)$ratio. Unfortunately, the $\mathrm{C}^{+} \lambda 1334$ absorption line is saturated and cannot be used directly to infer $N\left(\mathrm{C}^{+}\right)$. We therefore consider that the carbon metallicity can be derived from the silicon metallicity with some correction. Indeed, it is known that the metallicity of the $\alpha$-chain elements is enhanced compared to carbon by a factor of about two 22 when the mean metallicity is low (i.e. $\leq-1.0$ ). To be conservative, we consider that $Z(\mathrm{C})=Z(\mathrm{Si}) / 2$ and that silicon is not depleted into dust. Note that both assumptions maximize the $\mathrm{C}^{+*} / \mathrm{C}^{+}$ratio and, as a consequence, maximize the derived hydrogen density, $n_{\mathrm{H}}$. In addition, the very small $\mathrm{C}^{0} / \mathrm{C}^{+}$value shows that the ionization correction is negligible. Note also that absorptions due to $\mathrm{Si}^{3+}$ and $\mathrm{C}^{3+}$ are unusually weak in this damped system. The fit of the $\mathrm{C}^{+*} \lambda \lambda 1335.6,1335.7$ lines is shown in Fig. 2. As can be seen from this figure the observed $\mathrm{C}^{+*}$ profile suggests the presence of possible extra components. Our measured column density is therefore an upper limit which will turn into an upper limit on the 
hydrogen density. It must be noted that the strongest constraint comes from the 1335.6 line in the blue wing of the profile.

Results on the determination of $n_{\mathrm{H}}$ are presented in Figure 3. For a kinetic temperature in the range $85<T<285 \mathrm{~K}$, as suggested from the $\mathrm{H}_{2}$ absorption, we find $20<n_{\mathrm{H}}<35 \mathrm{~cm}^{-3}$ (solid curve on Fig. 3). All assumptions above maximize the hydrogen density which can therefore be considered for each temperature as a conservative upper limit.

\section{Cosmic Microwave Background radiation temperature}

The fine structure levels of $\mathrm{C}^{0}$ can be populated by several processes, mainly collisions with hydrogen atoms and electrons and pumping due to the local UV-radiation and to the CMBR. The different contributions can be estimated once the temperature and the particle density are known. We have derived above $85<T<285 \mathrm{~K}, 20<n_{\mathrm{H}}<35 \mathrm{~cm}^{-3}$ and $n_{\mathrm{e}} / n_{\mathrm{H}} \sim 0.001$.

Following Meyer et al. \& Roueff24, we investigate the fine-structure excitation of $\mathrm{C}^{0}$ keeping the particle density and the temperature within the ranges determined in the previous Sections. For a radiation field similar to that of the Milky-way25, the UV pumping rate from the ground state to the excited states of $\mathrm{C}^{0}$ is $7.55 \times 10^{-10} \mathrm{~s}^{-1}$. The corresponding value for the hydrogen collisional rate in the density and temperature ranges considered here is of the order of $\sim 1.2-1.4 \times 10^{-8} \mathrm{~s}^{-1}$. Thus the contribution due to UV pumping is negligible. Keenan et al.26 noticed that collisions with electrons are unimportant for kinetic temperatures in the range 100-500 K and a ratio of electron density to hydrogen density less than 0.01. Collisions with electrons can therefore be neglected.

The observed $2 \sigma$ range for $N\left(\mathrm{C}^{0 *}\right) / N\left(\mathrm{C}^{0}\right)$ and $N\left(\mathrm{C}^{0 * *}\right) / N\left(\mathrm{C}^{0}\right)$ are, respectively, $0.28-0.48$ and 0.020-0.060. Assuming collision by hydrogen atoms is the only process at work, we estimate the range of hydrogen density needed to explain the $\mathrm{C}^{0}$ populations. This density range is shown as a shaded region in Fig. 3. From this figure it is apparent that the observed upper limit on the hydrogen density derived above is not sufficient to populate the excited fine-structure levels of $\mathrm{C}^{0}$ and that an additional source of excitation is needed. The only process we are left with is direct 
pumping due to photons from the relic background radiation.

More generally, we estimate the allowed range for the CMBR temperature as a function of kinetic temperature, after taking into account all previously discussed processes. The allowed area in the $\mathrm{T}_{\mathrm{CMBR}}-\mathrm{T}_{\text {Kinetic }}$ plane is shown as the shaded region in Fig. 4 . This region is obtained using the upper limit on the hydrogen density derived above and the $2 \sigma$ ranges of the $\mathrm{C}^{0 *} / \mathrm{C}^{0}$ and $\mathrm{C}^{0 * *} / \mathrm{C}^{0}$ ratios. Thus the lower $\mathrm{T}_{\mathrm{CMBR}}$ boarder of this area gives a stringent lower limit on the CMBR temperature at $z_{\mathrm{abs}}=2.33771$.

Note that an upper limit on $\mathrm{T}_{\mathrm{CMBR}}$ is obtained assuming that the CMBR is the only excitation process at work. This limit is shown as a dotted line in Fig. 1 .

The presence of a relic radiation field at any redshift is more or less admitted by most of the practicing cosmologists. Its reality is proved observationally only in the local universe through direct measurements 327 and the Sunayev \& Zeldovich effect 28 . Our measurement demonstrates for the first time that the cosmic radiation exists at earlier times and has a higher temperature than today. In the standard Big-Bang cosmology the cosmic microwave background is a relic radiation left over from an early hot phase. In such a model the radiation evolves adiabatically in the expanding universe and the temperature at any redshift is simply $T(z)=T(z=0)(1+z)$. We summarise all the available upper limits in Fig. 5, together with our measurement. The prediction for the adiabatic expansion is shown as a dotted line. All the upper limits and our measurement are consistent with the predictions of the standard model. Doing similar analysis over a large redshift range will provide a direct and model independent measure of the evolution of the cosmic microwave background radiation. 


\section{References}

1. Alpher, R. A., Bethe, H. A. \& Gamov, G. Evolution of Chemical Elements. Phys. Rev., 73, 803-804 (1948)

2. Penzias, A. A. \& Wilson, R. . A measurement of excess antenna temperature at $4080 \mathrm{Mc} / \mathrm{s}$. Astrophys. J. 142, 419-421 (1965)

3. Mather, J. C. et al. Measurement of the cosmic microwave background spectrum by the COBE FIRAS instrument. Astrophys. J. 420, 439-444 (1994)

4. Bahcall, J. N., Joss, P. C., \& Lynds, R. On the Temperature of the Microwave Background Radiation at a Large Redshift. Astrophys. J. 182, L95-L98 (1973)

5. Meyer, D. M., Black., J. H., Chaffee, F. H., Foltz, C. B., \& York, D. G. An upper limit on the microwave background temperature at $z=1.776$. Astrophys. J. 308, L37-L41 (1986)

6. Songaila, A. et al. Measurement of the Microwave Background Temperature at a Redshift of 1.776. Nature 371, 43-45 (1994)

7. Songaila, A., Cowie, L. L., Hogan, C. \& Rugers, M. Deuterium Abundance and Background Radiation Temperature in High Redshift Primordial Clouds. Nature 368, 599-604 (1994)

8. Lu. L., Sargent, W. L. W., Womble, D. S. \& Barlow, T. A. Abundances at High Redshifts: The Chemical Enrichment History of Damped Lyman- $\alpha$ Galaxies. Astrophys. J. Suppl. Ser. 107, 475-520 (1996)

9. Jian Ge, Bechtold, J. \& Black, J. H. A New Measurement of the Cosmic Microwave Background Radiation Temperature at $z=1.97$. Astrophys. J., 474, 67-73 (1997)

10. Roth, K. C. \& Bauer, J. M. The z $=1.6748$ C I absorber toward PKS 1756+237, Astrophys. J. 515, L57-L60 
11. D'Odorico, S., Cristiani, S., Dekker, H., et al., Performance of UVES, the echelle spectrograph for the ESO VLT and highlights of the first observations of stars and quasars. Proc. SPIE Vol. 4005, 121-130 (2000)

12. Welty, D. E., et al. The diffuse interstellar cloud toward 23 Orionis. Astrophys. J. Suppl. Ser.124, 465-501 (1999).

13. Morton, D.C. Atomic data for resonance absorption lines. I - Wavelengths longward of the Lyman limit. Astrophys. J. Suppl. Ser. 77, 119-202 (1991)

14. Savage, B. D. \& Sembach, K. R. Interstellar Abundances from Absorption-Line Observations with the Hubble Space Telescope. Ann. Rev. Astron. Astrophys. 34, 279-330 (1996)

15. Pettini, M., Smith, L. J., King, D. L. \& Hunstead, R. W. The Metallicity of High-Redshift Galaxies: The Abundance of Zinc in 34 Damped Lyman- $\alpha$ Systems from $z=0.7$ to 3.4. Astrophys. J. 486, 665-680 (1997)

16. Ge, J. \& Bechtold J. $\mathrm{H}_{2}$ and $\mathrm{C}$ I in Damped Lyman- $\alpha$ Quasar Absorbers at Intermediate and High Redshifts. In eds. C.L. Carilli, S.J.E. Radford, K.M. Menten, G.I. Langston, Highly Redshifted Radio Lines, ASP Conf. Series Vol. 156, 121-131 (1999)

17. Shull, J. M., Tumlinson, J., Jenkins, E. B., et al. FUSE Observations of Diffuse Interstellar Molecular Hydrogen. astro-ph/0005014 (2000)

18. Jura, M. Interstellar clouds containing optically thick $\mathrm{H}_{2}$. Astrophys. J. 197, 581-586 (1975)

19. Dalgarno, A. \& Wright E. L., Infrared emissivities of $\mathrm{H}_{2}$ and HD, Astrophys. J., 174, L49-L51 (1972).

20. Srianand, R. \& Petitjean, P. Molecules in the $z_{a b s}=2.8112$ damped system toward PKS 0528-250. Astron. Astrophys. 335, 33-40 (1998)

21. Bahcall, J.N. \& Wolf, R.A. Fine-Structure Transitions. Astrophys. J. 152, 701-729 (1968) 
22. Timmes F, X., Lauroesch J. J., Truran J. W. Abundance histories of QSO absorption systems, Astrophys. J., 518, 468-476 (1995)

23. Nussbaumer, H. \& Rusca, C. Forbidden transitions in the C I sequence. Astron. Astrophys. 72, 129-133 (1979)

24. Launay, J. M. \& Roueff, E. Fine structure excitation of carbon and oxygen by atomic hydrogen impact. Astron. Astrophys. 56, 289-292 (1977)

25. Jenkins, E. B. \& Shaya, E. J. A survey of interstellar C I insight on carbon abundances UV grain albedoes, and pressures in the interstellar medium, Astrophys. J. 231, 55-72 (1979)

26. Keenan, F. P., Lennon, D., J., Johnson, C. T., \& Kingston, A. E, Fine structure population for the 2P ground states of C II, Mon. Not. R. astr. Soc., 220, 571-576 (1986)

27. Roth, K. C., Meyer, D. M. Cyanogen excitation in diffuse interstellar cloud. Astrophys. J. 441, $129-143(1995)$

28. Suniaev, R. A., Zeldovich, Ya. B. The velocity of clusters of galaxies relative to the microwave background - The possibility of its measurement. Month. Not. R. Astron. Soc. 190, 413-420 (1980).

AAS LATEX macros v4.0. 
Acknowledgments The observations presented here have been obtained using the Ultra-violet and Visible Echelle Spectrograph mounted on the $8.2 \mathrm{~m}$ KUEYEN telescope operated by the European Southern Observatory at Paranal, Chile. We gratefully acknowledge support from the Indo-French Centre for the Promotion of Advanced Research (Centre Franco-Indien pour la Promotion de la Recherche Avancée). PPJ thanks Andreas Kaufer and Merieme Chadid for their kind and efficient assistance at the telescope and IUCAA for hospitality during the time this work was completed. We thank T. Padmanabhan for useful comments. 
TABLE 1: Heavy elements from the damped Lyman- $\alpha$ system

$\begin{array}{ccccc}\text { Ion } & \log N\left(\mathrm{~cm}^{-2}\right) & b\left(\mathrm{kms}^{-1}\right) & {[\mathrm{Z} / \mathrm{H}]} & {[\mathrm{Z} / \mathrm{H}]-[\mathrm{Z} / \mathrm{H}] \odot} \\ & & & & \\ \mathrm{H}^{0} & 20.90 \pm 0.10 & \ldots & \ldots & \ldots \\ \mathrm{C}^{0} & 13.86 \pm 0.22 & 1.70 \pm 0.10 & \ldots . & \ldots . \\ \mathrm{C}^{0 *} & 13.43 \pm 0.07 & \ldots & \ldots & \ldots \\ \mathrm{C}^{0 * *} & 12.63 \pm 0.22 & \ldots & \ldots & \ldots \\ \mathrm{C}^{+*} & \leq 14.00 & \ldots & \ldots & \ldots \\ \mathrm{Mg}^{0} & 13.19 \pm 0.09 & \ldots . & & \ldots \\ \mathrm{Mg}^{+} & 15.44 \pm 0.09 & \ldots & -5.46 \pm 0.13 & -1.04 \pm 0.13 \\ \mathrm{Si}^{+} & 15.24 \pm 0.11 & \ldots & -5.66 \pm 0.19 & -1.20 \pm 0.10 \\ \mathrm{Fe}^{+} & 14.68 \pm 0.08 & \ldots . & -6.22 \pm 0.13 & -1.73 \pm 0.13\end{array}$

$N$ is the column density and $b$ the Doppler width of the line; $b=\left(2 k T / m+\mathrm{b}_{\mathrm{turb}}^{2}\right)^{1 / 2}$, where $m$ is the atomic mass, $T$ the temperature and $b_{\text {turb }}$ the characteristic turbulent velocity of the gas. $[\mathrm{Z} / \mathrm{H}]=\log N(\mathrm{Z})-\log N(\mathrm{H}$ I $)$ is the metallicity and $[\mathrm{Z} / \mathrm{H}]-[\mathrm{Z} / \mathrm{H}]_{\odot}$ the metallicity relative to the value measured in the Sun. We used the solar metallicities from Savage \& Sembach 14 . All wavelengths and oscillator strengths are from Morton 13 and Welty et al.1. 
TABLE 2: Fit results to rotational levels in the vibrational ground state of $\mathbf{H}_{2}$

$$
\begin{array}{cccc}
z_{\text {abs }} & \text { Level } & \log N\left(\mathrm{~cm}^{2}\right) & b\left(\mathrm{~km} \mathrm{~s}^{-1}\right) \\
& & & \\
2.33735 & \mathrm{~J}=0 & 3.60 \pm 0.34 \times 10^{15} & 24.07 \\
& \mathrm{~J}=1 & 3.34 \pm 0.40 \times 10^{15} & 24.07 \pm 10.20 \\
2.33754 & \mathrm{~J}=0 & 4.10 \pm 0.48 \times 10^{15} & 14.09 \\
& \mathrm{~J}=1 & 2.65 \pm 0.44 \times 10^{15} & 14.09 \pm 4.57 \\
2.33771 & \mathrm{~J}=0 & 2.30 \pm 1.00 \times 10^{16} & 4.62 \pm 0.36 \\
& \mathrm{~J}=1 & 8.30 \pm 2.60 \times 10^{16} & 4.62 \\
& \mathrm{~J}=2 & 1.59 \pm 0.27 \times 10^{16} & 4.62 \\
& \mathrm{~J}=3 & 9.39 \pm 1.10 \times 10^{15} & 4.62 \\
& \mathrm{~J}=4 & 4.42 \pm 0.80 \times 10^{14} & 4.62 \\
& \mathrm{~J}=5 & 4.64 \pm 0.77 \times 10^{14} & 4.62 \\
2.33793 & \mathrm{~J}=0 & 1.00 \pm 0.04 \times 10^{15} & 21.74 \\
\mathrm{~J}=1 & 6.69 \pm 0.40 \times 10^{15} & 21.74 \pm 1.40
\end{array}
$$



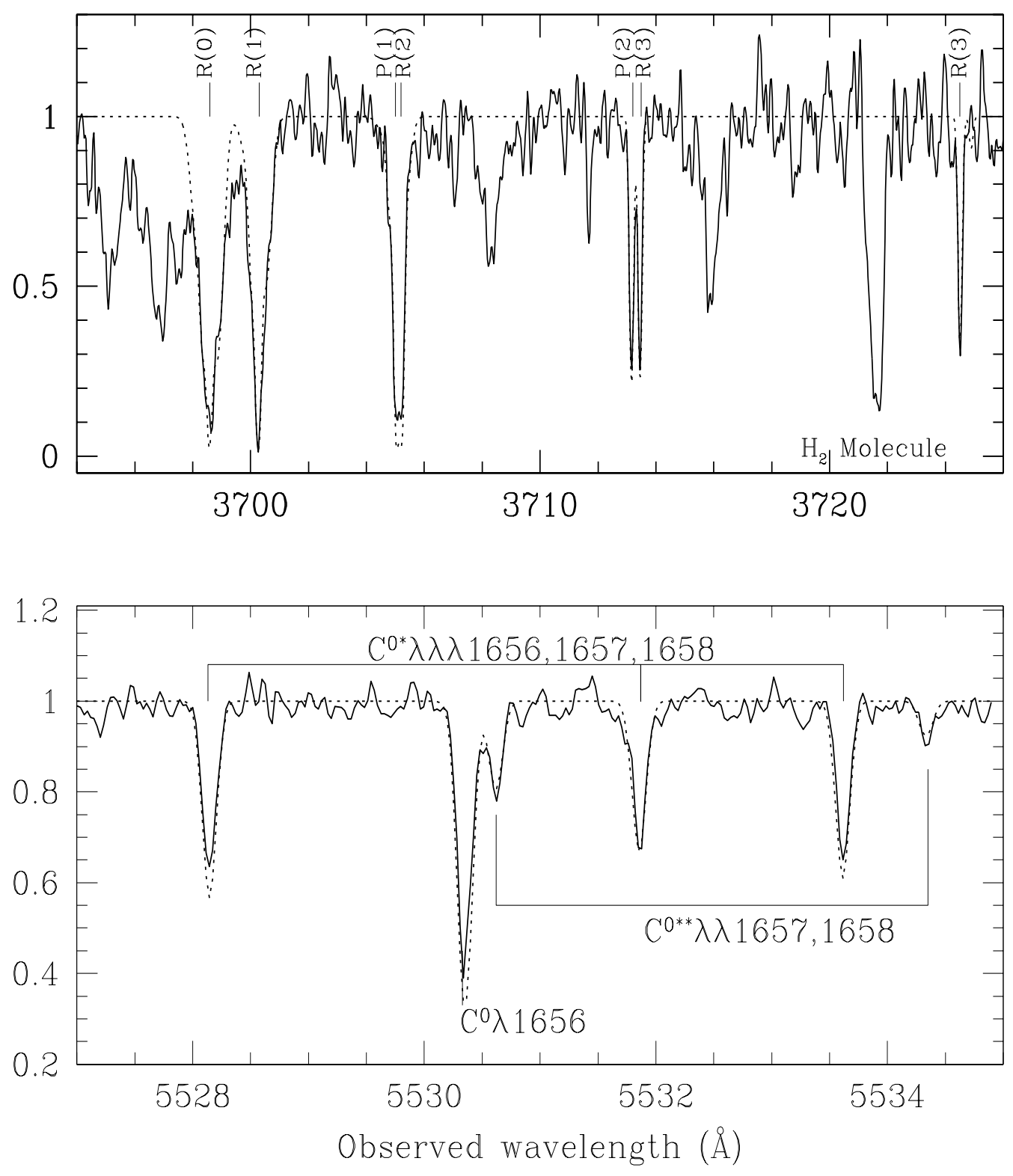

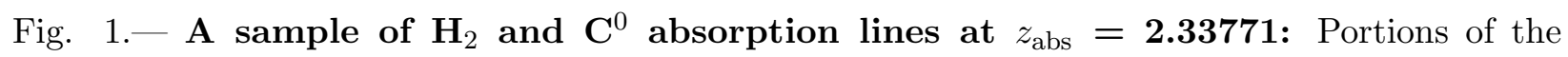
normalized spectrum of the quasar PKS 1232+0815 taken with the Ultra-violet and Visible Echelle Spectrograph mounted on the $8.2 \mathrm{~m}$ KUEYEN telescope of the European Southern Observatory on the Paranal mountain in Chile. Upper panel: A selection of $\mathrm{H}_{2}$ absorption lines from the $J=0$, 1, 2 and 3 rotational levels from the $\mathrm{v}=0-1$ Lyman band. The model fit with parameters given in Table 2 is overplotted to the data as a dashed line. Lower panel: Detection of absorption lines from $\mathrm{C}^{0}, \mathrm{C}^{0 *}$ and $\mathrm{C}^{0 * *}$ at $z_{\mathrm{abs}}=2.33771$ in the damped Lyman- $\alpha$ system. The model fit with parameters given in Table 1 is over-plotted to the data as a dashed line. 


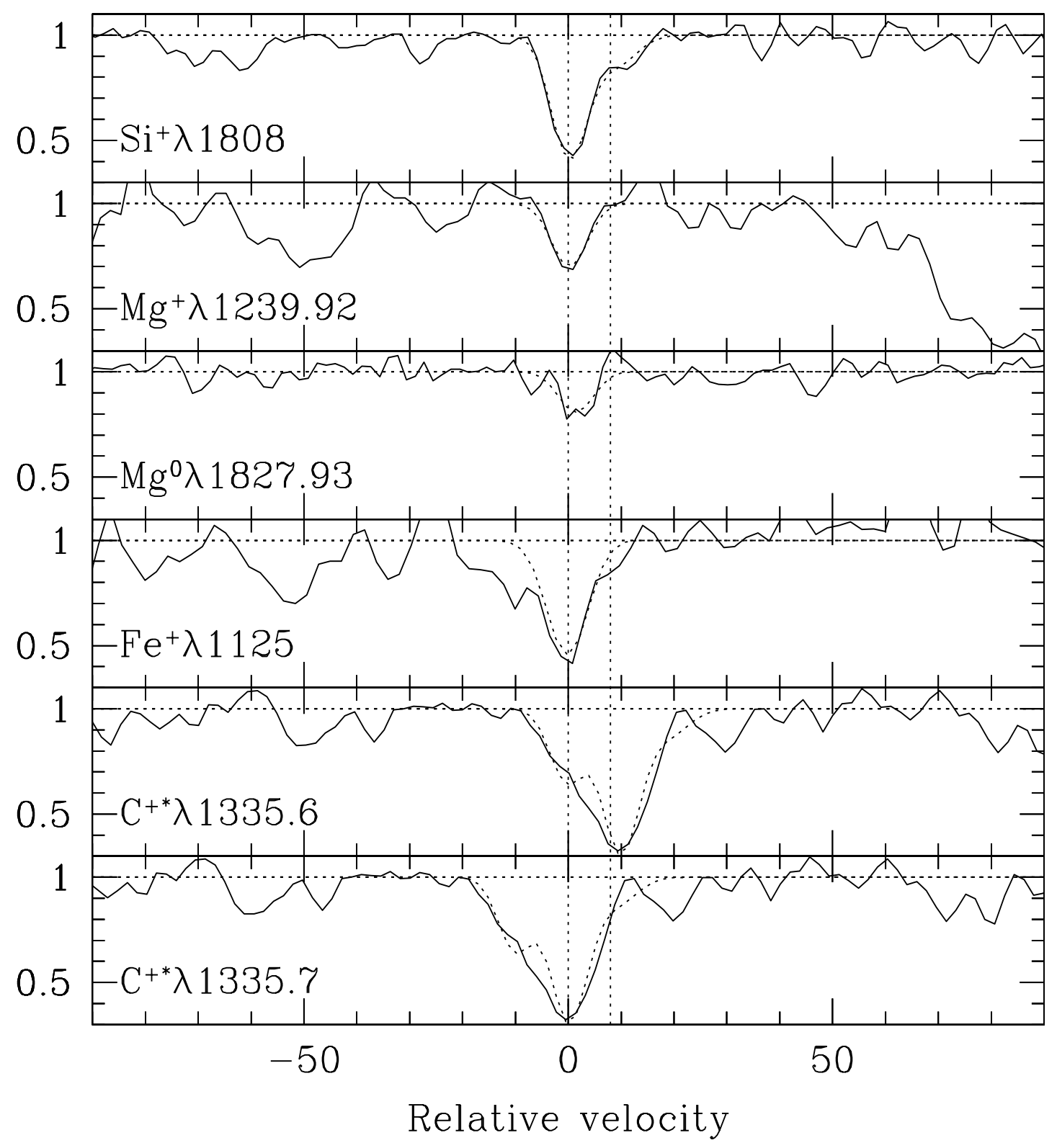

Fig. 2.- A sample of heavy element absorption lines at $z_{\mathrm{abs}}=2.33771$ : Absorption profiles of a few transitions (indicated on each panel) from the damped Lyman- $\alpha$ system toward PKS $1232+0815$. The normalized flux is given on a velocity scale with origin at $z=2.33771$. The $\mathrm{C}^{+*}$ profile is slightly broader than the other lines. The $\mathrm{C}^{+*}$ line is a blend of $\mathrm{C}^{+*} \lambda 1335.71$ and $\mathrm{C}^{+*} \lambda 1335.66$. Fitted models are over-plotted as dashed lines. The fit is performed over all the absorption lines available in the spectrum. Our best fitted column density of $10^{14} \mathrm{~cm}^{-2}$ for $\mathrm{C}^{+*}$ overpredicts the absorption at $\lambda 1335.6$. 


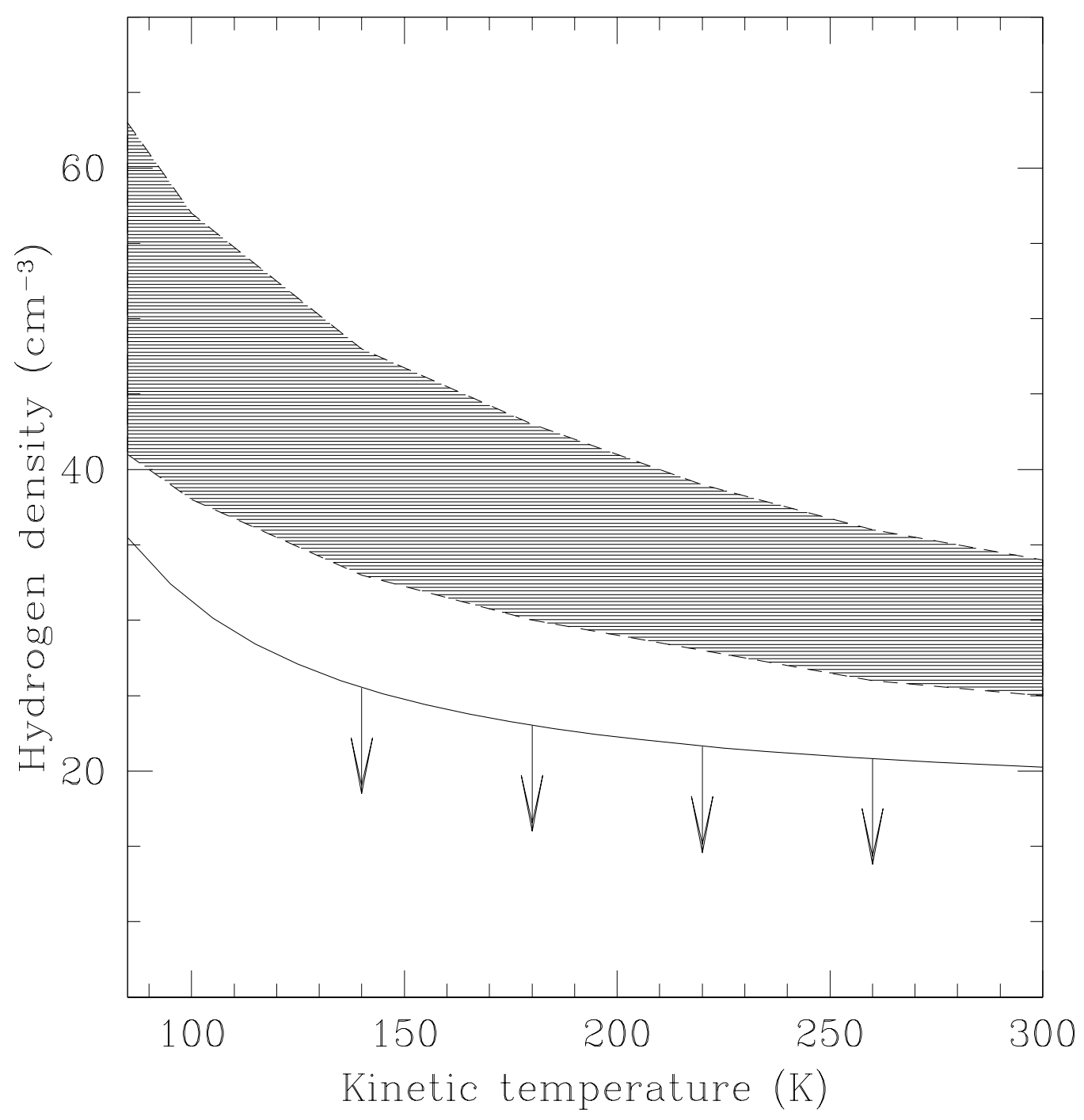

Fig. 3. - The hydrogen density as a function of kinetic temperature in the $z_{\mathrm{abs}}=\mathbf{2 . 3 3 7 7 1}$ cloud: The continuous curve with downward arrows shows the strict upper limit on the hydrogen density estimated from the fine-structure excitation of $\mathrm{C}^{+}$. The shaded area gives the $2 \sigma$ range for the hydrogen density required to explain the population ratios in the fine-structure levels of $\mathrm{C}^{0}$ assuming there is no cosmic background radiation. All other excitation processes (collisions by electrons and hydrogen atoms, UV pumping) have been taken into account. It is apparent that the observed density is too small to explain the $\mathrm{C}^{0}$ excitation demonstrating that the Cosmic Microwave Background exists at $z=2.33771$. 


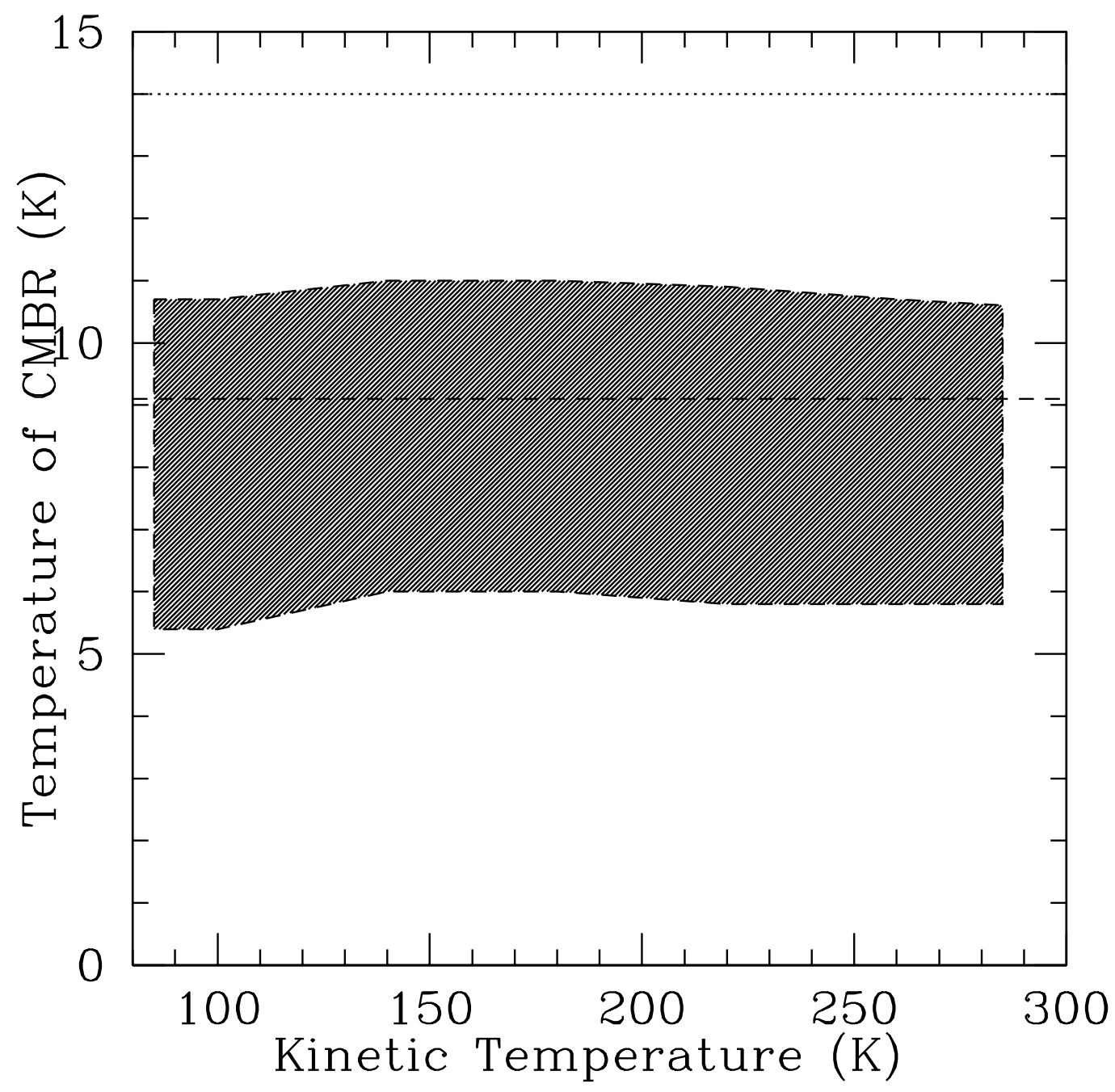

Fig. 4.- Cosmic microwave background temperature as a function of kinetic temperature of the gas: For a given temperature we obtain an upper limit of the hydrogen density from the $\mathrm{C}^{+}$fine-structure population. This is used to derive the contribution of hydrogen collisions to the $\mathrm{C}^{0}$ fine-structure excitation. The excess is used to determine the range for the CMBR temperature. The shaded region gives the $2 \sigma$ range of the Cosmic Microwave Background Radiation temperature allowed by the observed population ratios of neutral carbon fine-structure levels. The horizontal dotted line is the upper limit on $\mathrm{T}_{\mathrm{CMBR}}$ assuming $\mathrm{CMBR}$ to be the only excitation process. The dashed line is the predicted temperature at $\mathrm{z}=2.33771$ in the standard big-bang model. This demonstrates the presence of a background radiation with a temperature at least twice that measured in the local universe, $\mathrm{T}_{\mathrm{CMBR}, 0}=2.726 \mathrm{~K}$. 


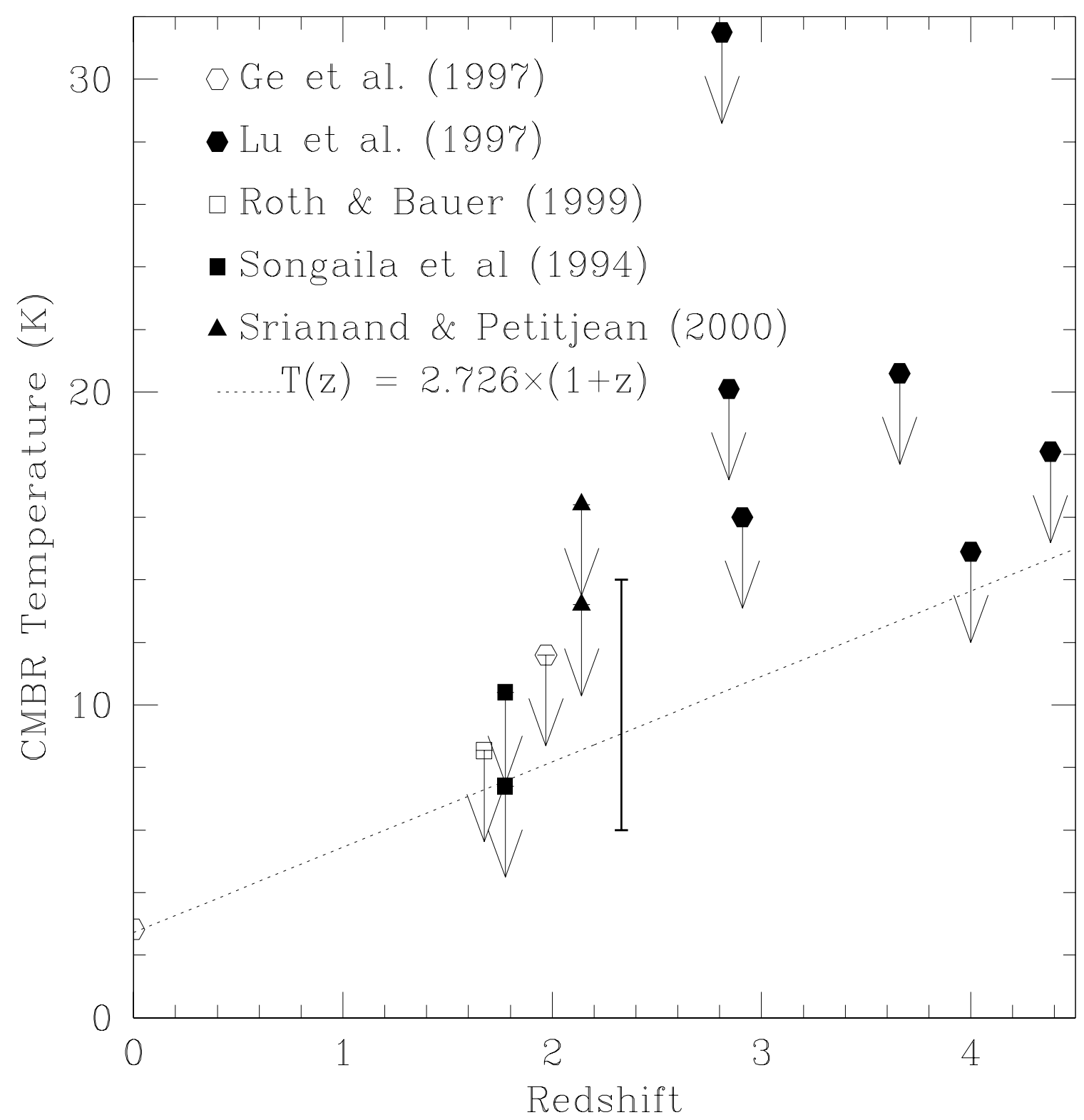

Fig. 5.- Measurements of the Cosmic Microwave Background Radiation temperature at various redshifts: The point at $z=0$ shows the result of the Cosmic Background Explorer $(\mathrm{COBE})$ determination $\mathrm{B}, T_{\mathrm{CMBR}}(0)=2.726 \pm 0.010 \mathrm{~K}$. Upper limits are previous measurements 6 , 国, e using the same techniques as in this paper. We also include our two new unpublished upper limits at $z=2.1394$ along the line of sight toward Tololo 1037-270. The measurement from this work, $6.0<T_{\mathrm{CMBR}}<14.0 \mathrm{~K}$ at $z=2.33771$, is indicated by a vertical bar. The dashed line is the prediction from the Hot Big Bang, $T_{\mathrm{CMBR}}(z)=T_{\mathrm{CMBR}}(0) \times(1+z)$. 